\title{
Intracranial complications of Serratia marcescens infection in neonates
}

\begin{abstract}
A Madide, J Smith
Ayanda Madide was employed as a paediatrician/neonatologist in the Division of Neonatology, Department of Paediatrics and Child Health, Tygerberg Children's Hospital, Stellenbosch University, Cape Town, South Africa. She relocated to the UK in 2015. Dr Madide's main interests include the provision of 'hands-on' clinical care and perinatal morbidity and mortality. Prof. Johan Smith is the clinical head of the neonatal intensive care unit and level III neonatal service of the Department of Paediatrics and Child Health at Tygerberg Children's Hospital. His main interests are neonatal pulmonology and surfactant development.
\end{abstract}

Corresponding author: J Smith (js7@sun.ac.za)

Even though Serratia marcescens is not one of the most common causes of infection in neonates, it is associated with grave morbidity and mortality. We describe the evolution of brain parenchymal affectation observed in association with S. marcescens infection in neonates. This retrospective case series details brain ultrasound findings of five neonates with hospital-acquired S. marcescens infection. Neonatal S. marcescens infection with or without associated meningitis can be complicated by brain parenchymal affectation, leading to cerebral abscess formation. It is recommended that all neonates with this infection should undergo neuro-imaging more than once before discharge from hospital; this can be achieved using bedside ultrasonography.

S Afr Med J 2016;106(4):342-344. DOI:10.7196/SAMJ.2016.v106i4.10206

Serratia marcescens infection has occurred sporadically from 2010 to 2014 in our 126-bed neonatal unit, with a tendency towards cluster infections. In each instance, staff at the Unit for Infection Prevention and Control, Tygerberg Hospital, Cape Town, South Africa employed their expertise to investigate, identify and manage appropriately the root cause of the cluster infection. On two separate occasions, colonised respiratory support equipment and suction points were identified as sources of infection; this situation was rectified. We recently noted a pattern of brain ultrasound findings consistently associated with the identification of $S$. marcescens septicaemia in neonates. These findings have not been noted in septicaemia secondary to other micro-organisms. The majority of neonates with such ultrasound findings died, despite intensive care and appropriate antimicrobial treatment having been instituted.

We describe a case series of five neonates with S. marcescens nosocomial infection in whom the abovementioned pattern was evident. In patient 1 , autopsy characterisation of these findings was done.

Ethical approval for the case series and a waiver of individual informed consent were granted by the Human Health Research Ethics Committee of Stellenbosch University, protocol number N15/08/068.

\section{Case 1}

Patient 1 was delivered by caesarean section because of maternal pre-eclamptic toxaemia and fetal affectation at an estimated 30 weeks of gestation (birth weight $1460 \mathrm{~g}$ ). Apart from antiretroviral, antihypertensive and antenatal corticosteroids medication, his mother had received no other treatment and had had a healthy pregnancy. He progressed well and was admitted to the neonatal high-care ward for further care because of a very low birth weight. He had a normal baseline full blood count and was treated with intravenous aminophylline and oral nevirapine for apnoea and HIV prophylaxis, respectively. He was commenced on formula feeds on his second day of life and required no respiratory support.

On day 6, he developed respiratory distress and progressed to hypoxic respiratory failure accompanied by convulsions, for which he had to be intubated and transferred to the neonatal intensive care unit (NICU) for ventilatory support. At this time, an infection screen was performed and he was commenced on meropenem and vancomycin antimicrobial therapy. A cranial ultrasound scan done on admission to the NICU showed a premature brain compatible with an estimated gestational age of 32 weeks, minimal vessel pulsation and predominant findings in the bilateral parieto-occipital area. The latter had welldefined hyperechogenicities with areas of cystic breakdown, predominantly on the left side. These findings were thought to represent ischaemic parenchymal injury and areas of white matter infarcts. The patient had a persistent low-voltage pattern on amplitudeintegrated electro-encephalography, remained in intractable hypotension and metabolic acidosis and succumbed to his illness within a day of admission to the NICU. His parents granted permission for an autopsy, and soon after he died, telephonic notification of Gramnegative bacilli on blood culture was received from the microbiology laboratory. This was later confirmed to be extended-spectrum betalactamase (ESBL)-producing S. marcescens.

Fig. 1 shows the predominant cranial ultrasound results of right parieto-occipital wedgeshaped hyperechogenicity in this patient; moderate lenticulostriate vasculopathy can also be

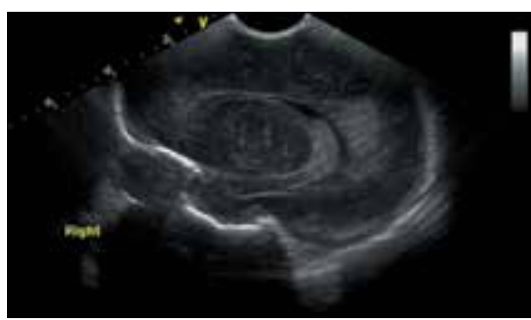

Fig. 1. Right parasagittal image.

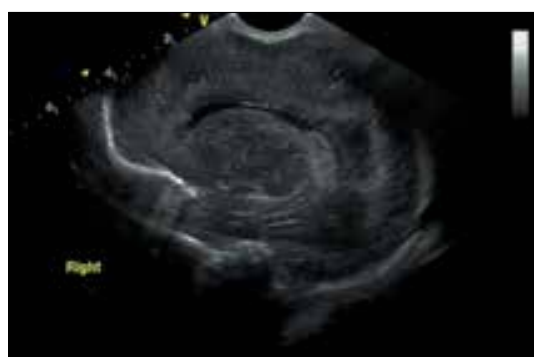

Fig. 2. Right parasagittal image approximately 12 hours later. 
seen. Cranial ultrasonography was performed through the anterior fontanelle with an $8 \mathrm{MHz}$ curvilinear probe using a Vivid S5 General Electric Healthcare ultrasound machine (USA).

Fig. 2 shows progressive change to cystic breakdown of the same area approximately 12 hours later.

In Fig. 3, the beginning of cystic breakdown within a hyperechogenic area is noted in the left posterior parietal region.

Autopsy findings included extensive white matter necrosis of the temporal lobes, haemorrhagic infarction of temporal and occipital lobes, leptomeningitis in sections of the parietal cortex, and haemorrhagic necrosis of the cerebellum. The brainstem was also noted to be disintegrated.

\section{Cases 2 - 5}

Details of four other patients with similar clinical and microbiological findings are given in Table 1, and the associated cranial ultrasound findings can be seen in Fig. 4 .

The images of patient 2 were obtained with an $8 \mathrm{MHz}$ sector probe using a Vivid S5 General Electric Healthcare ultrasound

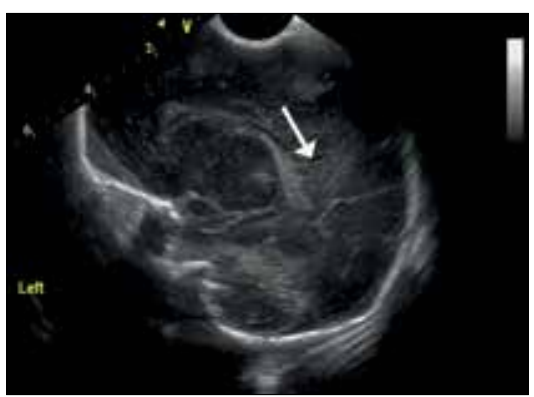

Fig. 3. Left parasagittal image. machine. The images of patients 3,4 and 5 were obtained using a $10 \mathrm{MHz}$ sector probe (Antares Sonoline Siemens Medical Solutions, USA).

\section{Discussion}

S. marcescens is an Enterobacteriaceae micro-organism that is widespread in the environment, but not a common component of the human faecal flora. Most infections are acquired exogenously and spread in and among hospitals. ${ }^{[1]}$ Hand-held transmission appears to be the primary mechanism of nosocomial spread. Environmental sources in hospitals have been found to include suction taps, colonised disinfectants or soap, and inadequately sterilised breast pumps and respirators. Disease in humans has been associated with infection of the urinary tract, respiratory tract, local wounds and central vein catheters. Colonisation in neonatal units has been associated with up to a tenfold increase in the rate of $S$. marcescens bacteraemia and meningitis. ${ }^{[2]}$ Significant brain injury caused by ventriculitis, brain abscesses or porencephalic cysts is described in the majority of infants with meningitis. In our neonatal unit, we have noted intracranial complications in the presence or absence of meningitis.

NICUs in developed countries have reported serial infection outbreaks with this micro-organism over the past decade, although not all of these focused primarily on associated neurological morbidity. In a recently published report from Italy, ${ }^{[3]}$ sepsis and pneumonia were the most severe clinical features described, with a mortality rate of $7 \%$. Only 1 of 18 affected neonates was found to have meningitis and a brain abscess. This neonate survived. ${ }^{[3,4]}$ Published reports on neonatal $S$. marcescens infection in developing countries are limited with regard to availability and content. A global report on causes of neonatal sepsis in developing countries cited Serratia species as the fifth most common cause, accounting for $0.5 \%$ and $0.3 \%$ of early and late neonatal sepsis, respectively. ${ }^{[5]}$ In their report on bloodstream infections in neonates in a developing country over a 12-month period, Ballot et al. ${ }^{[6]}$ do not mention $S$. marcescens as one of the causative organisms. Unpublished data from our institution detailing infection patterns in very-low-birth-weight infants over an 18-month period, found that ESBLproducing $S$. marcescens accounted for $12 \%$ of late-onset neonatal sepsis. S. marcescens and ESBL-producing $S$. marcescens accounted for $5 \%$ and $8 \%$, respectively, of infectionrelated mortality over a 24 -month period in our unit.

Published case reports describe diagnosis of brain abscesses after an initial diagnosis of $S$. marcescens septicaemia and meningitis. ${ }^{[7,8]}$ In our unit, three babies on whom cerebrospinal fluid (CSF) analysis had not been done died within 24 hours of onset of illness. Of the two survivors we describe, one had abnormal CSF analysis results of high protein, pleiocytosis and no organisms on culture, while the other had normal CSF results on two separate occasions. In both instances, the lumbar puncture and CSF analysis were done only after the diagnosis of septicaemia and commencement of

Table 1. Summary of details of patients with Serratia marcescens

\begin{tabular}{|c|c|c|c|}
\hline Patient details & $\begin{array}{l}\text { Age at Serratia } \\
\text { diagnosis }\end{array}$ & Cranial ultrasound findings & Outcome \\
\hline $\begin{array}{l}\text { Case } 2 \\
\text { GA } 29 \text { wks } \\
\text { BW } 1608 \mathrm{~g} \\
\text { Positive }\end{array}$ & $\begin{array}{l}6 \text { days } \\
\text { CUS on day } 6\end{array}$ & $\begin{array}{l}\text { Minimal cerebrovascular pulsation. Right-side moderate lenticulostriate } \\
\text { vasculopathy. Bilateral extensive frontoparietal well-circumscribed } \\
\text { hyperechogenicity with areas of echolucent breakdown }\end{array}$ & Died on day 6 \\
\hline $\begin{array}{l}\text { Case } 3 \\
\text { GA } 31 \text { wks } \\
\text { BW } 1370 \mathrm{~g} \\
\text { Negative }\end{array}$ & $\begin{array}{l}7 \text { days } \\
\text { CUS on day } 7\end{array}$ & $\begin{array}{l}\text { Bilateral frontoparietal parenchymal hyperechogenicity with areas of echolucent } \\
\text { cystic breakdown. Bilateral basal ganglia/thalamic hyperechogenicity }\end{array}$ & Died on day 7 \\
\hline $\begin{array}{l}\text { Case } 4 \\
\text { GA } 33 \text { wks } \\
\text { BW } 1550 \mathrm{~g} \\
\text { Positive }\end{array}$ & $\begin{array}{l}9 \text { days } \\
\text { CUS on days } 9 \text {, } \\
11 \text { and } 16\end{array}$ & $\begin{array}{l}\text { Initial bilateral anterior and posterior irregular hyperechogenicity. Progressed } \\
\text { to echolucent cystic breakdown } 48 \text { hours later and to well-circumscribed thick- } \\
\text { walled lesions with central echolucency } 7 \text { days later - suggestive of abscess } \\
\text { formation }\end{array}$ & Alive on discharge \\
\hline $\begin{array}{l}\text { Case } 5 \\
\text { GA } 26 \text { wks } \\
\text { BW } 840 \mathrm{~g} \\
\text { Negative }\end{array}$ & $\begin{array}{l}6 \text { days } \\
\text { CUS on days } 33 \\
\text { and } 47\end{array}$ & $\begin{array}{l}\text { Bilateral frontoparietal and parieto-occipital well-circumscribed thick-walled } \\
\text { echolucent cystic lesions suggestive of abscess formation }\end{array}$ & Alive on discharge \\
\hline
\end{tabular}




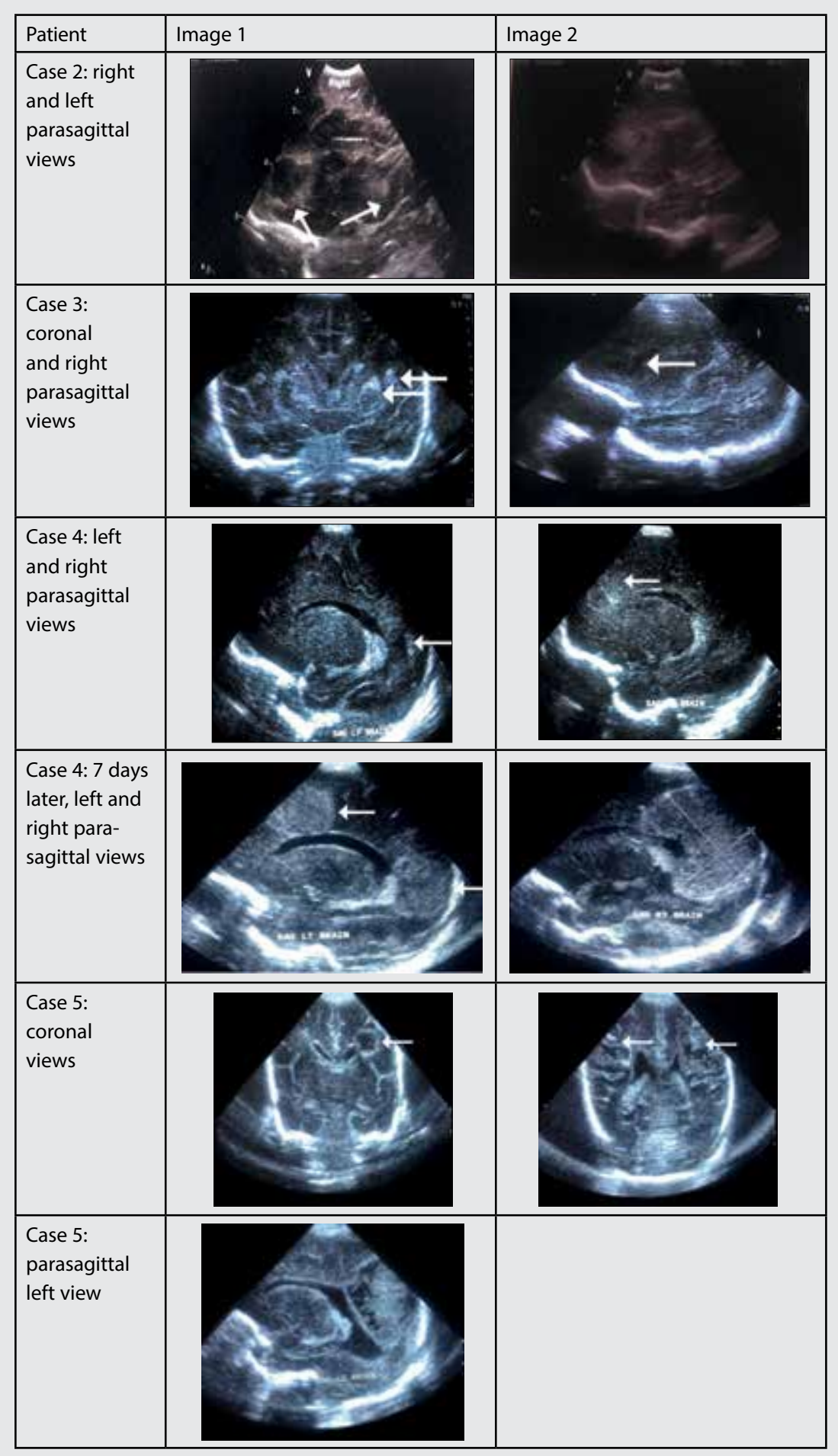

Fig. 4. Patient cranial ultrasound images.

antibiotic therapy. With the exception of only one patient, we describe changes in the brain before the diagnosis of Serratia infection had been made in the babies with acute onset of illness. We also describe the progression and changing pattern sizes of these thick-walled cysts tend to remain the same on serial imaging. We postulate that the initial changes represent areas of ischaemic injury, which progress to infarction and liquefaction, and that the cysts represent abscess formation within the brain parenchyma.

We conclude that in a neonate who suddenly and unexpectedly becomes ill, cranial ultrasonography should be an urgent additional investigation. If changes such as inhomogeneous hyperechogenicities are noted, brain affectation by a serious bacterial infection such as $S$. marcescens should be considered and appropriate antibiotic cover instituted without delay. Follow-up imaging within $24-48$ hours and again within 7 and 14 days in survivors will aid in identifying progression of injury and planning for neurosurgical intervention and rehabilitation. We suggest that cranial ultrasonography should be performed more than once before discharge from hospital in all neonates diagnosed with Serratia septicaemia, regardless of the presence or absence of neurological symptoms or an abnormal CSF analysis. This is based on our subsequent observations of intracranial complications, including abscess formation in neurologically asymptomatic neonates with normal CSF findings, as in the case of patient 5 . We also propose that good-quality bedside neuro-imaging can be undertaken with ultrasonography as often as clinically required in these critically ill neonates, without the need to move them from the NICU.

1. Donnenberg MS. Enterobateriaceae. In: Mandell GL, Bennett JE, Dolin R, eds. Principles and Practice of Infectious Diseases, vol 2.7th ed Philadelphin, USA: Churchill Livingstone Elrevier, 2010.
20.

2. Gruber WC, Fisher RG. Serratia. In: Feigin RD, Cherry JD, eds. Feigin and Cherry Textbook of Paediatric Infectious Diseases. vol. 1. 4th ed. Philadelphia, USA: Saunders, 1998.

3. Casolari C, Pecorari M, Della Casa E, et al. Serratia marcescens in a neonatal intensive care unit: Two long-term multiclone outbreaks in a 10-year observational study. New Microbio 2013;36:373-383.

4. Samuelsson A, Isaksson B, Hanberger H, Olhager E. Late-onse neonatal sepsis, risk factors and interventions: An analysis of recurrent outbreaks of Serratia marcescens, 2006 - 2011. J Hosp Infect 2014;86:57-63. DOI:10.1016/j.jhin.2013.09.017

5. Zaidi AKM, Thaver D, Ali SA, Khan TA. Pathogens associated with sepsis in newborns and young infants in developin countries. Pediatr Infect Dis J 2009.28.S10-S18.

6. Ballot DE, Nana T, Sriruttan C Cooper PA. Bacterial bloodPed Pediatrics 2012;2012:508512. DOI:10.5402/2012/508512

7. Hirooka MT, de Vasconcellos Fontes RB, Diniz EM, Pinto FC, Matushita H. Cerebral abscess caused by Serratia marcescens in a Matushita $\mathrm{H}$. Cerebral abscess caused by Serratia marcescens in premature neonate. Arq Neuropsiquiatr 2007;65(4-A):1018-1021. 8. Messerschmidt A, Prayer D, Olischar M, Pollak A, Birnbacher R. Brain abscesses after Serratia marcescens infection on a neonatal intensive care unit: Differences on serial imaging. Neuroradiology 2004;46(2):148-152.

Accepted 23 October 2015 